\title{
Operación Descentralizada en Grandes Sistemas de Potencia usando Algoritmos Genéticos
}

\author{
Francisco J. Vargas $^{(1) *}$, Rosa E. Correa ${ }^{(1)}$ y Juan M. Ramírez ${ }^{(2)}$ \\ (1) Facultad de Minas, Universidad Nacional de Colombia, Sede Medellín, Carrera 80 No. 65 - 31 , \\ Medellín-Colombia (e-mail: jvargas@xm.com.co, recorrea@unal.edu.co). \\ (2) Centro de Investigación y de Estudios Avanzados del IPN-Unidad Guadalajara, Av. del Bosque 1145 , \\ Col. el Bajío, Zapopan, 45019, Jalisco-México (e-mail: jramirez@gdl.cinvestav.mx).
}

${ }^{*}$ Autor a quien debe ser dirigida la correspondencia

Recibido Jul. 3, 2013; Aceptado Jul. 31, 2013; Versión final recibida Sep. 12, 2013

\begin{abstract}
Resumen
Este artículo propone una metodología para la coordinación y operación de grandes sistemas de potencia de forma descentralizada, simplificando la optimización y reduciendo los tiempos de cómputo. La búsqueda óptima del voltaje de los generadores, cambiador de tomas, y compensación, se logra utilizando una técnica evolutiva, planteándola como un problema de optimización multiobjetivo. Se considera la descomposición tanto de la red como el problema de optimización y se valida con dos casos de prueba (39 y 190 nodos). Los resultados muestran una operación óptima global preservando la estabilidad de voltaje y la gestión de intercambios entre áreas, con mínima información operativa, simplicidad en los equivalentes y reducción en tiempos de cómputo. Los resultados son similares a los obtenidos mediante una estrategia de optimización centralizada.
\end{abstract}

Palabras clave: sistemas de potencia, operación descentralizada, descomposición de redes, optimización multiobjetivo, algoritmos genéticos

\section{Decentralized Operation in Large Power Systems using Genetic Algorithms}

\begin{abstract}
This paper proposes a methodology based on decentralized operation and coordination of large power systems, obtaining the system's optimization in a simplified way, and reducing time consumption. The optimal search of generator voltage, tap changer and compensation, is accomplished by using an evolutionary technique, and considering the problem as a multi-objective optimization case. In this paper, both the decomposition network and the optimization problem are applied to two study cases (39 and 190 buses). Global optimal operation is attained, preserving the voltage stability and the exchanges among areas, with minimal operational information, simplicity in the equivalents and time consuming reduction. Results are similar to those obtained by a centralized optimization strategy.
\end{abstract}

Keywords: power systems, decentralized operation, networks decomposition, multi-objective optimization, genetic algorithms 


\section{INTRODUCCIÓN}

En los últimos años, se ha observado un incremento de las interconexiones entre Múltiples Operadores de Red (MTSO) debido a la apertura de mercados eléctricos y la integración de varias redes eléctricas, como es el caso de la red Europea y la norteamericana. Estos sistemas están caracterizados por una alta dimensionalidad, altos costos de las TIC, celos en el manejo de la información operativa, conflictos de intereses, y descoordinación entre MTSO. Estas situaciones impactan la operación por inestabilidades y apagones, afectando el suministro de energía (Venkatesh et al., 2000; SFOE, 2003; FERC, 2005). Por esto, el operador del sistema independiente (OSI), quien coordina y opera cada sistema, tiene el desafío de mantener la integridad operativa de la red de manera confiable, segura y económica.

Así, la optimización es una herramienta utilizada en sofisticados esquemas de control, como la coordinación de las acciones de control entre áreas interconectadas (Phulpin, 2009 y Phulpin et al 2010). En este mismo sentido, se han propuesto estrategias centralizadas, tanto para el control del voltaje y gestión de potencia reactiva (López, 2013, Mousavi y Cherkaoui, 2011), como para la congestión de intercambios (Arnold et al, 2007). Sin embargo, estas estructuras no han sido lo suficientemente flexibles, y han tenido dificultades a la hora de una implementación.

Por otro lado, con el objeto de facilitar la operación, coordinación y análisis entre MTSO se ha propuesto dividir el problema de optimización global en subproblemas y descomponer la red eléctrica. Ahora en cuanto a la descomposición de la red se utilizan equivalentes, los cuales son determinantes en el desempeño del método de optimización (Phulpin et al, 2010). En estos equivalentes, normalmente los intercambios entre áreas interconectadas se modelan como una carga adicional (Biskas y Bakirtzis 2006), (Kim y Baldick, 1997). La problemática radica en que la gran mayoría de los equivalentes requieren la creación de nodos ficticios y nuevas variables de acople por cada enlace.

En cuanto a la descomposición del problema de optimización global en subproblemas, se han presentado varias técnicas de solución. Se ha utilizado Programación Lineal (LP) enfocando mediante el problema primal y dual de la LP (Dantzig y Wolfe, 1960). Asimismo, otras como las condiciones de Karush-KuhnTucker (KKT) de primer orden (Biskas y Bakirtzis, 2006), multiplicadores de Lagrange asociados con las restricciones complicantes (Nogales et al 2003), multiplicadores de Lagrange aumentada, y para la relajación de las variables de acople, mediante el principio del problema auxiliar (APP) (Marinakis et al 2007) o empleando el método del subgradiente (X. Wang et al, 2001). La limitación de estas propuestas es el empleo de técnicas convencionales de optimización, donde se restringe a la maximización o minimización de un solo objetivo y se soluciona mediante procesos iterativos.

En las referencias consultadas no se encuentra una metodología sencilla que minimice los tiempos de respuesta para estimar los puntos óptimos de operación de un gran sistema de energía, donde interactúan MTSO, donde existen altos volúmenes de información y, sobre todo, un alto riesgo de que se presenten inestabilidades e incluso apagones generalizados. En este artículo se propone una estrategia de operación descentralizada, a partir de la descomposición, no solo de la red eléctrica, sino también del problema de optimización, empleando una técnica heurística y evolutiva, "Nondominated Sorting Genetic Algorithm" (NSGA-II) multiobjetivo, cuya solución sea adecuada y que el cálculo se logre en el menor tiempo posible, en comparación con una posible estrategia centralizada.

\section{DESCOMPOSICIÓN DE LA RED ELÉCTRICA}

Para la descomposición de la red eléctrica se asume el criterio del menor número de enlaces; la manera natural como las redes se interconectan entre países o regiones. Sean las áreas $i$ y $j$, para $i, j=1,2, \ldots, N$; siendo $N$ el número de áreas de control independientes que integran la red eléctrica, el nodo $A$ pertenece al área $i$ y el nodo $C$ pertenece al área $j$, mientras que el nodo $B$ corresponde a la frontera entre ambas áreas $i$ y $j$, respectivamente. Se propone que el flujo de potencia activa y reactiva por las líneas de interconexión $A B$ y $B C$ entre las áreas $i$ y $j$, correspondan a un equivalente $P Q$ para un área $i$ y un equivalente $P V$ para el área $j$, reflejados en el nodo frontera B, Fig. 1. Estos equivalentes se denominan equivalentes generalizados.

Una vez calculados los equivalentes generalizados, se procede a modificar el arreglo de la restricción de igualdad $\hat{h}_{i}^{k}\left(x_{T S O i}, u_{T S O i}, Z_{T S O i}^{k}{ }^{*}\right)=0$, presente en el problema del flujo óptimo de potencia (OPF), ecuaciones (3)-(5). Para un área cualquiera $i$, el equivalente $P Q$ en una iteración $\mathrm{k}$ del flujo de potencia puede descomponerse en parte real e imaginaria de la siguiente manera: 
$\operatorname{real}\left(V_{i, A_{i} B_{j}}^{k} \cdot \operatorname{conj}\left(I_{i, A_{i} B_{j}}^{k}\right)\right)-Z P_{i, A_{i} B_{j}}^{k}=0$

$$
\operatorname{imag}\left(V_{i, A_{i} B_{j}}^{k} \cdot \operatorname{conj}\left(I_{i, A_{i} B_{j}}^{k}\right)\right)-Z Q_{i, A_{j} B_{j}}^{k}=0
$$

donde $V_{i, A i B j}^{k}$ y $l_{i, A i B j}^{k}$ son los voltajes y corrientes en los extremos de la interconexión $A_{i} B_{j}$ del área $i$ en la iteración $k$. Los parámetros $Z P_{i, A_{i} B_{j}}^{k}$ y $Z Q_{i, A_{i} B_{j}}^{k}$, equivalen al intercambio de potencia programado por el respectivo enlace, que es sustituido por un equivalente $P Q$ en el nodo $B$. De forma análoga se procede para el área $j$, en el cual la restricción de igualdad se actualiza con el equivalente $P V$. De ésta manera se satisface la ecuación $S_{A B}=-S_{B A}$.

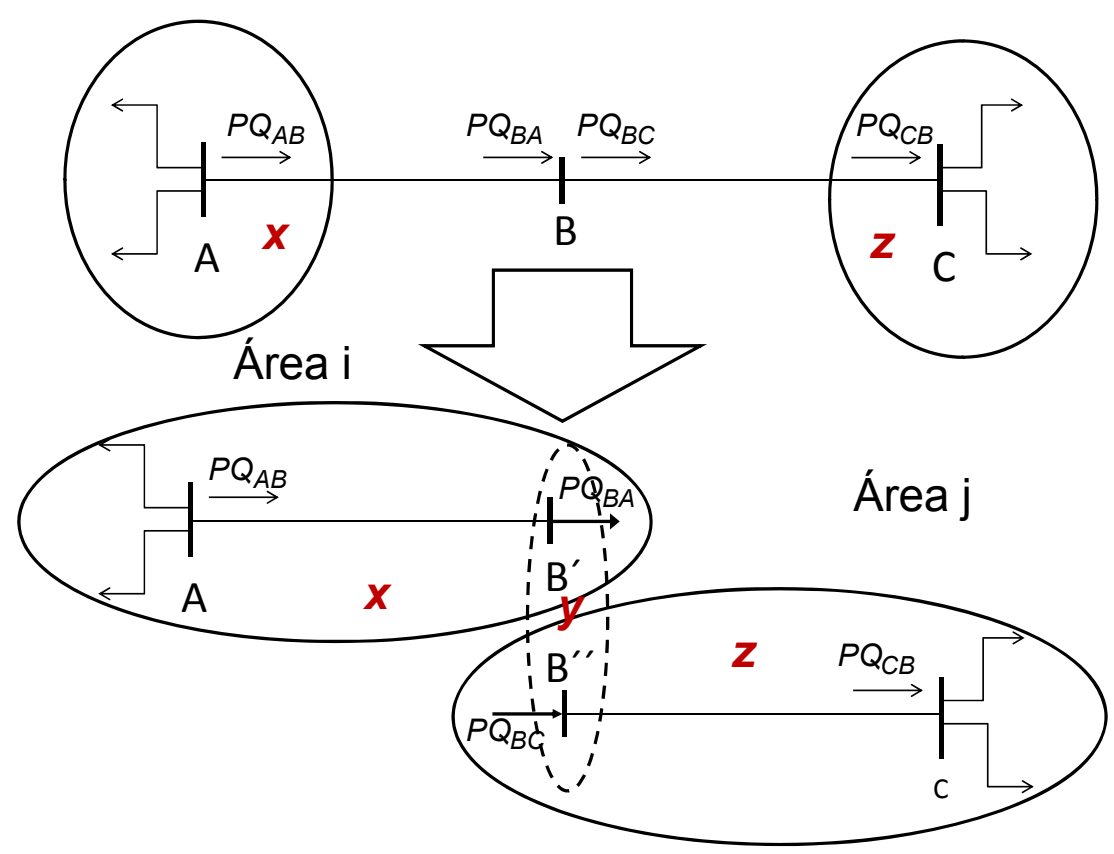

Fig. 1: Equivalentes $P Q$ y $P V$ generalizados para las áreas i y j

Por otro lado, una vez dividida la red en áreas de control independientes, utilizando el modelo equivalente generalizado, se procede a la descomposición del OPF general en subproblemas empleando una formulación de un problema multiobjetivo por cada área de control. A continuación se formula el problema de optimización multiobjetivo.

\section{OPTIMIZACION DEL PROBLEMA MULTIOBJETIVO EN UN SISTEMA ELÉCTRICO}

Un problema de optimización multiagente (por cada área de control) y a la vez multiobjetivo (OPFMO) se define como la tarea de minimizar (o maximizar) $F(\vec{x})=\left(f_{1}(\vec{x}), f_{2}(\vec{x}), \ldots, f_{k}(\vec{x})\right)$ sujeto a $g_{i}(\vec{x}) \leq 0, i=\{1,2, \ldots, m\}$ restricciones de desigualdad y $h_{j}(\vec{x})=0, j=\{1,2, \ldots, p\}$ restricciones de igualdad con $\vec{x} \in \Omega$. Una solución a este problema minimiza (o maximiza) las componentes del vector $F(\vec{x})$ donde $\vec{x}$ es un vector $\mathrm{n}$-dimensional de variables de decisión $\vec{x}=\left(x_{1}, x_{2}, \ldots, x_{n}\right)$ de algún universo $\Omega$ (Coello et al, 2007). Las restricciones $g_{i}(\vec{x}) \leq 0$ y $h_{j}(\vec{x})=0$ deben ser satisfechas al mismo tiempo. $F(\vec{x})$ y $\Omega$ contiene todos los posibles $\vec{x}$ que pueden ser usados para satisfacer la evaluación de $F(\vec{x})$. El vector de variables de decisión puede ser continuo o discreto mientras las $k$ funciones, pueden ser lineales o no, y continuas o discretas. La función de evaluación $F=\Omega \rightarrow \Lambda$ es una transformación del vector de variables de decisión $\vec{x}=\left(x_{1}, x_{2}, \ldots, x_{n}\right)$ en un vector de respuestas $\vec{y}=\left(a_{1}, a_{2}, \ldots, a_{k}\right)$. Cuando el problema es OPFMO el significado de óptimo debe ser redefinido, ya que la presencia de varios objetivos en conflicto puede ocasionar que la mejora en alguno de ellos provoque el deterioro de otros. Por lo tanto, un problema de OPFMO consiste en encontrar el mejor compromiso (balance) entre esos objetivos, denominado óptimo de Pareto. 
Aplicando el anterior concepto a un sistema eléctrico, éste puede fraccionarse mediante equivalentes de red, preservando su propia identidad, atributos y características. La Figura 2 muestra la partición de una red en dos áreas de control independiente: El Operador del Sistema de Transmisión (TSO) 1 y TSO 2, donde cada área dispone de la información del intercambio de potencia con el subsistema vecino, lo que permite optimizar el sistema de manera autónoma velando por su propia economía y seguridad.

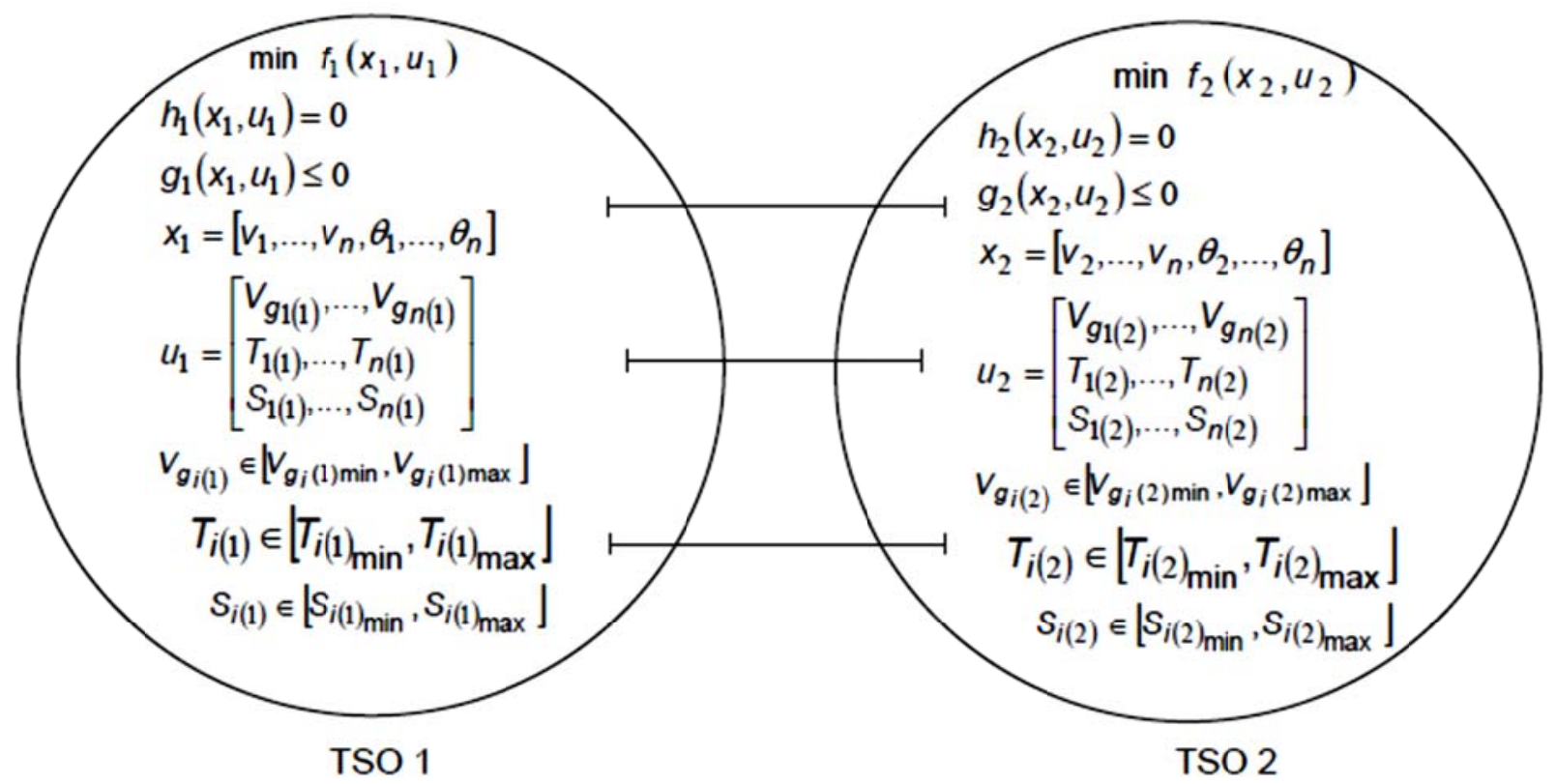

Fig. 2: Sistema fraccionado en dos subáreas TSO1 y TSO2

La formulación del OPFMO, se describe como

$\min F O_{i}(x)$

sujeto a

$h(x, u)=0$

$g(x, u) \leq 0$

donde, $\mathrm{FO}_{i}$ : representa las funciones objetivo, que para el presente trabajo se asumen la minimización de: $F O_{1}$, pérdidas activas, $F O_{2}$ pérdidas reactivas y $F O_{3}$ índice de estabilidad de voltaje $(L-$ index); $x$ y $u$, las variables de estado y de control a optimizar. El vector $x=[\theta, V]$, contiene los ángulos $\left(\theta_{i}\right)$ y magnitudes de los voltajes $\left(V_{i}\right)$ de todos los nodos del sistema; el vector $u$ representa las variables de control que se van a optimizar, estas corresponden al voltaje terminal de los generadores $\left(V_{g i}\right)$, el cambiador de tomas de los transformadores $\left(T_{i}\right)$, la compensación paralela conectable $\left(S_{i}\right) ; h(x, u)$ representa las restricciones de igualdad (el balance de potencia activa y reactiva), $g(x, u)$ representa las ecuaciones de desigualdad correspondientes a las restricciones de las variables de decisión $\left(V_{g i}, T_{i}, S_{i}\right)$.

Las funciones objetivo consideradas en el presente trabajo están evaluadas de la siguiente manera. Para una línea sencilla, las pérdidas de potencia activa $F O_{1}$ y reactiva $F O_{2}$, están dadas por las siguientes expresiones:

$$
\begin{aligned}
& F O_{1}=\sum_{i}^{n l} P_{M W_{i}} \quad \text { con } P_{M W_{i}}=R_{i} l_{i}^{2}=R_{i} \frac{\left(V_{s i}-V_{r i}\right)^{2}}{x_{i}^{2}} ! \\
& F O_{2}=\sum_{i}^{n l} Q_{V A R_{i}} \quad \text { con } Q_{V A R_{i}}=X_{i} l_{i}^{2}=X_{i} \frac{\left(V_{s i}-V_{r i}\right)^{2}}{X_{i}^{2}}
\end{aligned}
$$

donde $X_{i}$ es la reactancia de línea, $R_{i}$ es la resistencia de línea, (l) es la corriente de línea, $V_{s}$ y $V_{r}$ corresponden al voltaje en los extremos de la línea, $P_{M W_{i}}$ y $Q_{V A R i}$ son las pérdidas de potencia activa y 
reactiva, $\left(n_{l}\right)$ es el número total de líneas. Por otro lado, la estabilidad de voltaje puede ser estimada por medio de índices de estabilidad (Suganyadevia y Babulal, 2009), que para el presente trabajo se asume $L$ index, que puede además evaluar dos aspectos: 1) estima el margen de estabilidad de todos los nodos del sistema, 2) señala el nodo más débil de todo el sistema. Este índice toma valores $0 \leq L$-index $\leq 1$, donde el valor cero significa (sistema sin carga) y uno (colapso de voltaje). Este índice corresponde al objetivo $\mathrm{FO}_{3} \mathrm{y}$ se calcula por la siguiente expresión:

$\mathrm{FO}_{3}=L-$ index $=\max _{j \in \alpha_{L}}\left|1-\frac{\sum_{i \in \alpha_{G}} F_{j i} V_{i}}{V_{j}}\right|$

donde, $\alpha_{L}$ es el vector de los nodos de carga, $\alpha_{G}$ es el vector de los nodos de generación, $F_{i j}$ es la submatriz de la matriz híbrida $H, V_{i}$ y $V_{j}$ corresponden a los voltajes de nodo, respectivamente; para más detalle ver (Kessel y Glavitisch, 1986).

\section{Restricciones}

Las restricciones de igualdad (ecuación (4)), corresponden a las ecuaciones del flujo de potencia que para un nodo cualquiera resultan,

$$
\begin{aligned}
& P_{G_{i}}-P_{D_{i}}+P_{i}(V, \theta)=0 \text { para } i=1,2, \ldots, n_{1} ! \\
& ! \\
& Q_{G_{i}}-Q_{D_{i}}+Q_{i}(V, \theta)=0 \text { para } i=1,2, \ldots, n_{2} !
\end{aligned}
$$

donde $n_{1}$ es el número de nodos del sistema, excepto el nodo de holgura, $n_{2}$ todos los nodos del sistema, $P_{G i}$ y $Q_{G i}$ son la potencia activa y reactiva del generador $i$-th, $P_{D i}$ y $Q_{D i}$ son la carga activa y reactiva del $i$-th nodo de carga, y $P_{i}(V, \theta), Q_{i}(V, \theta)$ son la potencia activa y reactiva inyectada en el $i$-th nodo, respectivamente.

De igual manera, las restricciones de desigualdad (ecuación (5)), reflejan las limitaciones operativas y físicas de las variables de estado y control que se optimizan. En la formulación del OPFMO se consideran las siguientes restricciones límites de potencia activa, reactiva y voltaje terminal de los generadores: $P_{G i-m i n} \leq P_{G i} \leq P_{G i-m a x} ; Q_{G i-m i n} \leq Q_{G i} \leq Q_{G i-m a x}$ y $V_{G i-m i n} \leq V_{G i} \leq V_{G i-m a x}$, donde $P_{G i-m i n}$ y $P_{G i-m a x}$, son los límites mínimo y máximo de potencia activa, $Q_{G i-\min }$ y $Q_{G i-m a x}$, de potencia reactiva, $V_{G i-m i n}$ y $V_{G i-m a x}$, del voltaje terminal para el generador $i$-th; límites de la posición del cambiador de tomas bajo carga de los transformadores, definidos por: $T_{i-\min } \leq T_{i} \leq T_{i-\max }$ donde $T_{i}$ es la posición del tap del transformador $i$-th, $T_{i-\text { min }}$ y $T_{i-m a x}$ corresponden a la posición mínima y máxima, respectivamente; límites de la compensación paralela conectable tanto, inductiva como capacitiva en el nodo $i$-th, definidos por: $S_{i-m i n} \leq S_{i} \leq S_{i-m a x}$, donde $S_{i}$ es la compensación, $S_{i-\min }$ y $S_{i-\max }$ corresponden a los valores mínimo y máximo, respectivamente. Para el presente trabajo se asumen $T_{i}$ y $S_{i}$ son variables continuas. En este trabajo, por la naturaleza del problema OPFMO no-lineal se emplean técnicas heurísticas evolutivas, como es el caso también de compensación reactiva (Borges et al, 2012), restauración del servicio en redes de distribución, (Mendoza y Rojas, 2009), mostrando un mejor desempeño que otras técnicas similares (Amarnath y Ramana, 2011).

El algoritmo NSGA-II, fue propuesto para resolver tres problemas que se presentan en el algoritmo NSGA original (Srinivas y Deb, 1994) la ausencia de elitismo, el ordenamiento no dominado, y la dependencia del parámetro de partición (López, 2013). Las características principales del NSGA-II son: el ordenamiento no dominado elitista, usando una población auxiliar que reduce la complejidad de los chequeos de dominancia, la preservación de diversidad calculando distancia de apilamiento, la asignación de "un valor de aptitud", que se basa en rangos de no dominancia y que incorpora los valores de distancia de apilamiento. Deb et al (2002a) plantean una búsqueda concurrente usando división de dominio en el NSGA-II, los autores sugieren guiar la búsqueda a diferentes secciones del frente de Pareto, transformando los objetivos mediante una suma ponderada que modifica el concepto de dominancia y permite que los procesos se enfoquen en diferentes regiones de búsqueda. Asimismo, introducen un operador de migración para posibilitar la cooperación entre procesos.

La técnica del Algoritmo Genético de Múltiples objetivos (AG-MO), se puede resumir en las siguientes fases.

1) Inicializar la población: La población es inicializada aleatoriamente con ( $p o p$ ) individuos y cada individuo corresponde a un cromosoma, integrado por las variables de decisión. En el presente artículo son: voltaje 
terminal de los generadores $\left(V_{g i}\right)$, el cambiador de tomas de los transformadores $\left(T_{i}\right)$, la compensación paralela conectable $\left(S_{i}\right)$, todas ellas variables continuas.

2) Calcular la aptitud de cada individuo: En este proceso se determina la aptitud o dominancia de un individuo dentro de la población. La clasificación de no dominancia de los individuos se lleva a cabo con los individuos que no son dominantes, quienes conforman el primer arreglo que estarán al frente denominado $\left(F_{1}\right)$ y se les asigna un rango $\left(r_{1}\right)$ de uno, a medida que los individuos van siendo dominantes conforman otro frente $\left(F_{i}\right)$ y se les va asignando un rango $\left(r_{i}\right)$ mayor a uno, y así sucesivamente. Por otro lado a cada frente conformado por individuos no dominantes se les asigna una distancia de apilamiento, dada por la ecuación (11), la cual calcula la distancia Euclidiana entre dos puntos basados en las $m$ funciones objetivos del espacio hiper dimensional $m$. Estos dos valores, rango y distancia de apilamiento se adicionan al vector fila cromosoma $[x]$,

$d_{i}=\left|\frac{f_{m}^{\left(I_{i+1}^{m}\right)}-f_{m}^{\left(I_{i-1}^{m}\right)}}{f_{m}^{\max }-f_{m}^{\min }}\right|$

3) Generar una nueva población a partir de los mejores individuos. Este procedimiento se logra comparando dos soluciones, que poseen dos atributos, rango de no dominancia $\left(r_{i}\right)$, según el frente de Pareto y la distancia local de apilamiento $\left(d_{i}\right)$. La selección retorna la solución ganadora (i) basándose en dos criterios fundamentales: si tiene mejor rango: $r_{i}<r_{j}$, y si tiene el mismo rango pero $(i)$ tiene mejor distancia de apilamiento: $d_{i}>d_{j}$.

4) Ejecutar la operación de cruzamiento y mutación de individuos. Este proceso consiste en reunir un conjunto de soluciones padres y descendientes que se obtienen por medio de los operadores de cruce y mutación. El algoritmo usa el algoritmo "Simulated Binary Crossover" (SBX), considerando la probabilidad de cruzamiento $(p c=0.9)$ y la mutación polinómica con una probabilidad de mutación $(p m=1 / n)$, donde $(n)$ es el número de variables de decisión.

5) Regresar al paso 2) hasta que se cumpla un criterio de convergencia, (Deb, 2002; Srinivas, 1994).

En la siguiente sección se presenta una metodología multiobjetivo para grandes sistemas eléctricos descentralizados. En la siguiente sección se presenta una metodología multiobjetivo para grandes sistemas eléctricos descentralizados.

\section{METODOLOGÍA PROPUESTA}

Los sistemas descentralizados ofrecen mayor autonomía y agilidad en la operación a los diversos operadores de la red. En esta metodología se explotan estos atributos mediante la descomposición tanto de la red eléctrica como la del problema de optimización. Se propone una optimización multiobjetivo, que permite tomar en cuenta diferentes aspectos de la operación, pérdidas activas, pérdidas reactivas y estabilidad de voltaje, y la reducción de los tiempos de cálculo al involucrar menos variables en el proceso y simplicidad en los equivalentes de red.

Por la complejidad y diversidad de las variables involucradas en el proceso de optimización se propone la solución utilizando una técnica heurística evolutiva, tal como algoritmos genéticos, con la cual se logra una convergencia muy rápida a puntos de operación adecuados, en la región óptima. A partir de un despacho económico se fijan las variables de frontera, evitando así la multiplicidad de cálculos de los intercambios entre áreas independientes.

Se incluye la seguridad de la red al tener en cuenta un índice de estabilidad, L-index, como una de las funciones objetivo. Este aspecto permite determinar los rangos admisibles de las diferentes variables.

Para seleccionar los valores más adecuados dentro de los valores admisibles dados por un frente de Pareto, se utilizan el valor mínimo y la mediana del conjunto de soluciones.

En la validación de la metodología se comparan intercambios, pérdidas y tiempos de cálculo con los resultados obtenidos al aplicar una estrategia centralizada.

La metodología se resume en los siguientes siete pasos y en la Figura 3. 


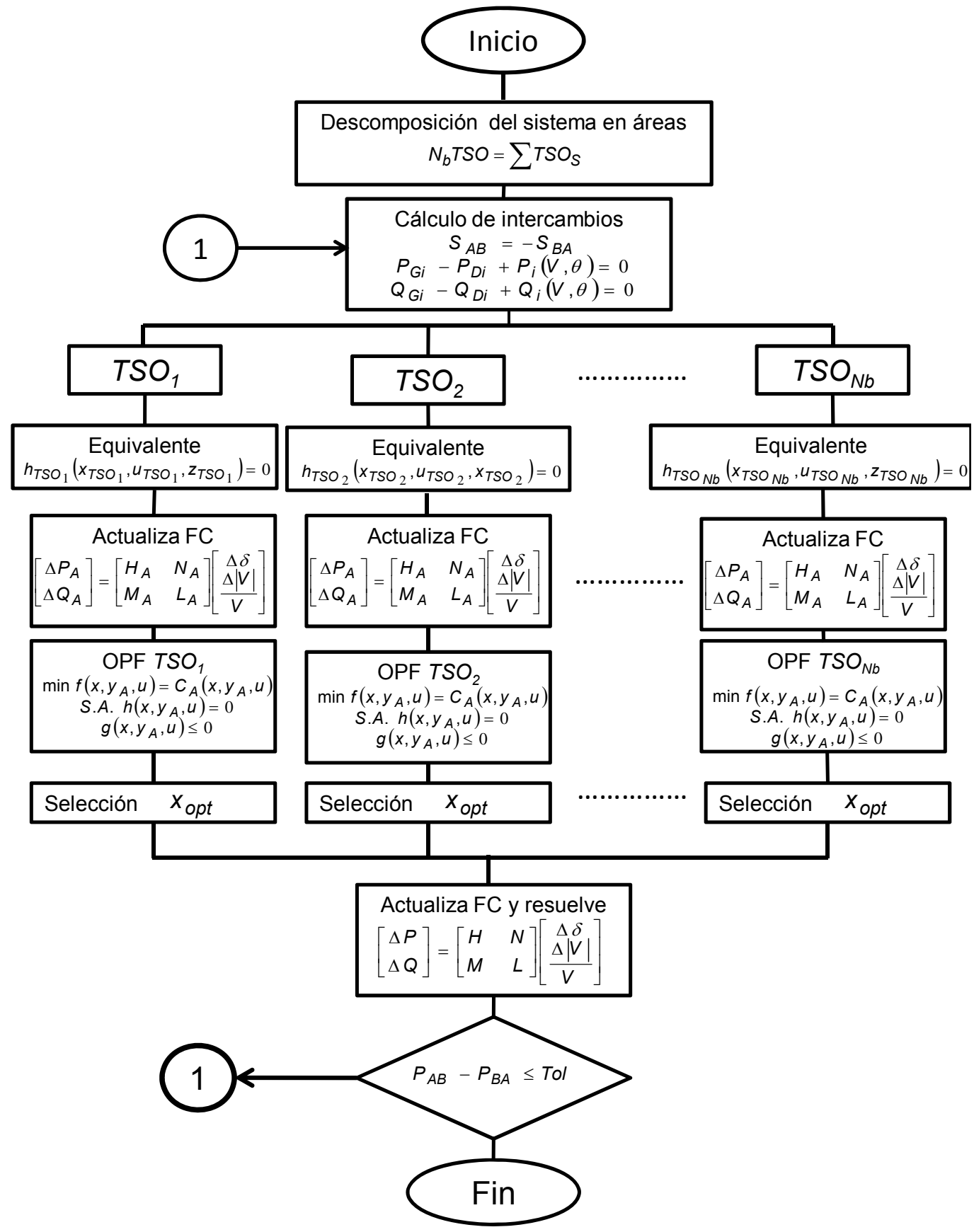

Fig. 3: Metodología OPFMO propuesta

1.- Se debe descomponer la red de energía eléctrica en áreas de control independientes, el cual se lleva a cabo considerando el menor número de enlaces entre ellas.

2.- A partir de un despacho económico y un flujo de potencia se fijan los intercambios entre las áreas, cuyos valores son la base para calcular los equivalentes de la red eléctrica y se consideran constantes durante todo el proceso.

3.- Se calculan los equivalentes generalizados. Estos equivalentes se construyen para cada área de control, considerando modelos $P Q$ y $P V$ en cada enlace entre áreas.

4.- Se construye el modelo para el flujo óptimo de potencia, multiobjetivo (OPFMO) para cada área de control, con base en los equivalentes propuestos.

5.- Se soluciona el problema de optimización utilizando una técnica heurística evolutiva, NSGA-II, calculando los mejores vectores que conforman el frente de Pareto, correspondiente a las variables de control: Voltaje en terminales de los generadores, $V_{g i}$, cambiadores de tomas, $T_{i}$ y la compensación, $S_{i}$. 
6.- Del frente de Pareto, se selecciona el mejor vector que en este contexto se considera el vector óptimo $u^{*}$, con base en el análisis estadístico del valor mínimo y la mediana de todas las soluciones obtenidas en el OPFMO, según el siguiente procedimiento:

$$
\min =\mid \sum\left(\text { Mediana }\left(f_{i}\right)-\min \left(f_{i}\right)\right) \mid \operatorname{para} i=1,2, \ldots, n_{f}
$$

donde, Mediana $\left(f_{i}\right)$ es la mediana del conjunto de valores obtenidos para la función objetivo $f_{i}$, min $\left(f_{i}\right)$ corresponde al menor valor obtenido para $f_{i}$ y $n_{f}$ el número de funciones objetivos consideradas en el problema de optimización multiobjetivo.

7.- Finalmente, se evalúa el flujo de potencia para la red completa considerando las variables del vector óptimas $u^{*}$ de cada área de control.

Esta metodología se propone como una buena estrategia para ser utilizada en la operación de grandes redes de energía eléctrica, en áreas autónomas, con intercambio de información mínima y con un buen desempeño en lo relacionado con los tiempos de cálculo. En la siguiente sección se presenta la validación de la metodología en una red de 39 nodos y una de 190 nodos.

\section{CASOS DE ESTUDIO}

Las redes consideradas corresponden a una red de 39 nodos, que es un equivalente modificado del sistema de energía inglés (Padiyar, 1996) y la red de 190 nodos, correspondiente a un equivalente de la red Mexicana de energía (González et al, 2011). Con el fin de realizar el análisis se definen los siguientes referentes.

Caso base: En este caso se tiene el cálculo de los intercambios de la red completa, sin optimización, asumiendo un despacho económico con flujo de potencia.

Caso centralizado: Se calcula del flujo óptimo de potencia, en la red con una configuración Centralizada, considerando los intercambios definidos en el caso base

Caso descentralizado. Se calcula del flujo óptimo de potencia, en la red con una configuración Descentralizada, considerando los intercambios definidos en el caso base, optimizando cada una de las áreas independientes, resolviendo el mismo problema de optimización, con las particularidades de cada área.

Con el objetivo de valorar el desempeño de los diferentes casos, se define el siguiente criterio de comparación. Se considera que los resultados son satisfactorios si se tiene una tolerancia en intercambios y pérdidas de potencia activa menor al $5 \%$, fundamentado en valores admisibles en una operación real (caso Colombiano).

\section{Aplicación a la red de 39 nodos}

La red está compuesta por 39 nodos, 10 generadores, 29 líneas, 4 enlaces de interconexión, 12 transformadores, 4 cambiadores de tomas de transformadores, localizados entre los nodos: 32-33, 31-32, 06-22 y 10-12, y 4 elementos de compensación, ubicados en los nodos $32,35,36$ y 37. Las variables de control $\quad\left(\left(V_{g i}\right), \quad\left(S_{i}\right), \quad\left(T_{i}\right)\right) \quad$ están acotadas así: $\quad-2.0 p . u \leq S_{i} \leq 2.0 p . u$ para $i=1, \ldots, 4$, 0.75 p.u $\leq \operatorname{Tap}_{i} \leq 1.25$ p.u para $i=1, \ldots, 4$ y 0.90 p.u $\leq V_{g i} \leq 1.1$ p.u para $i=1, \ldots, 10$. A continuación se sigue el procedimiento de la metodología:

Paso 1: Partición de la red, considerando el criterio de mínimos enlaces la red se divide en tres áreas independientes, la cual se muestra en la Figura 4.

Paso 2: Se calculan los flujos de potencia y el flujo entre áreas, Figura 5.

Paso 3: Cálculo de equivalentes de red. Estos equivalentes generalizados, se forman a partir de los enlaces y el resultado del flujo de potencia, Tabla 1.

Paso 4: Se construye el modelo del OPFMO. Con base en los equivalentes se actualiza el OPF para cada área. Las funciones objetivos consideradas son: $F O_{1}$ reducción de pérdidas activas y $F O_{2}$ reducción de pérdidas reactivas en las líneas de transmisión; y $\mathrm{FO}_{3}$ minimización del índice de estabilidad de voltaje $L$ - 
index. Las variables de decisión corresponden a: voltaje terminal generadores $\left(V_{g i}\right)$, total 10 ; la potencia reactiva de los elementos de compensación estáticos $\left(S_{i}\right)$, total 4 ; y el cambiador de tomas de transformadores $\left(T_{i}\right)$, total 4 , respectivamente.

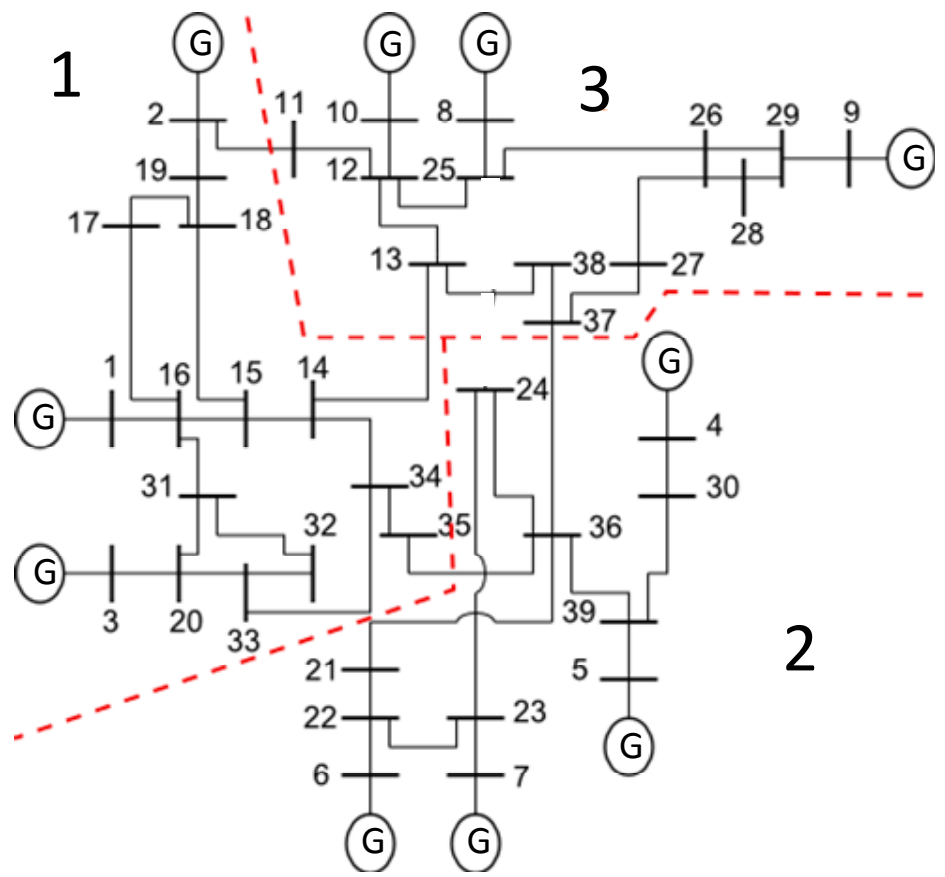

Fig. 4: División del Sistema de 39 nodos en 3 TSOs

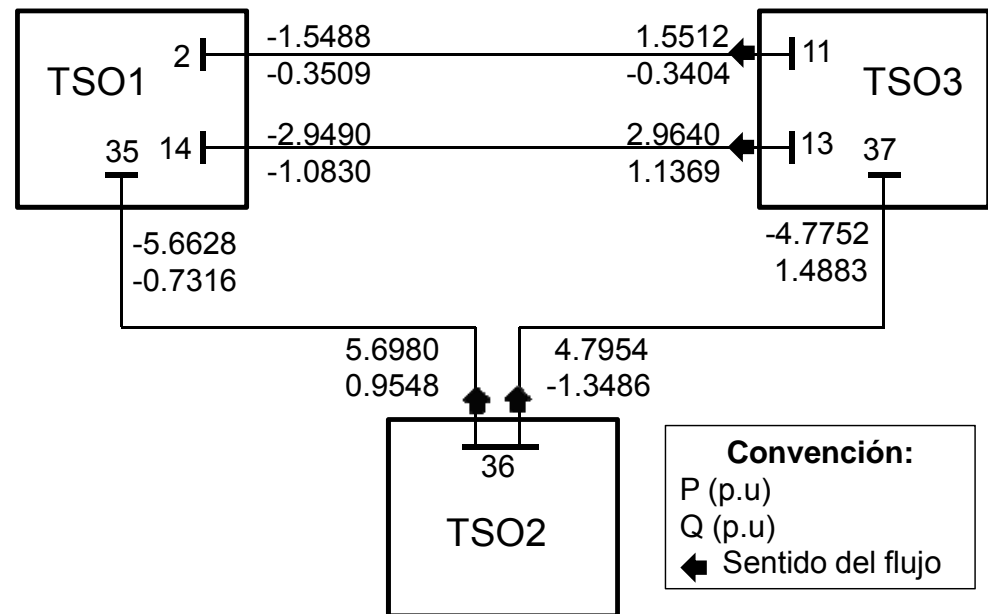

Fig. 5: Intercambios programados entre los 3 TSOs

Tabla 1: Equivalentes generalizados del sistema de 39 nodos

\begin{tabular}{|c|c|c|c|c|c|c|}
\hline \multirow{2}{*}{ TSO } & \multirow{2}{*}{ Nodo } & \multirow{2}{*}{ Línea } & & \multicolumn{3}{|c|}{ Equivalente } \\
\cline { 4 - 7 } & & & Nodo $\mathrm{F}$ & $\mathrm{Tipo}$ & $\mathrm{P}(\mathrm{p} . \mathrm{u})$ & $\mathrm{Q}(\mathrm{p} . \mathrm{u})$ \\
\hline \multirow{4}{*}{1} & 2 & $\mathrm{~L} 11-2$ & 11 & $\mathrm{PV}$ & 1.5512 & -0.3404 \\
\cline { 2 - 7 } & 14 & $\mathrm{~L} 13-14$ & 13 & $\mathrm{PV}$ & 2.9640 & 1.1369 \\
\cline { 2 - 7 } & 35 & $\mathrm{~L} 36-35$ & 36 & $\mathrm{PV}$ & 5.6980 & 0.9548 \\
\hline \multirow{3}{*}{2} & 36 & $\mathrm{~L} 35-36$ & 35 & $\mathrm{PQ}$ & -5.6628 & -0.7316 \\
\cline { 2 - 7 } & 36 & $\mathrm{~L} 37-36$ & 37 & $\mathrm{PQ}$ & -4.7752 & 1.4883 \\
\hline \multirow{3}{*}{3} & 11 & $\mathrm{~L} 2-11$ & 2 & $\mathrm{PQ}$ & -1.5488 & -0.3509 \\
\cline { 2 - 7 } & 13 & $\mathrm{~L} 14-13$ & 14 & $\mathrm{PQ}$ & -2.9490 & -1.0830 \\
\cline { 2 - 7 } & 37 & $\mathrm{~L} 36-37$ & 36 & $\mathrm{PV}$ & 4.7954 & -1.3486 \\
\hline
\end{tabular}




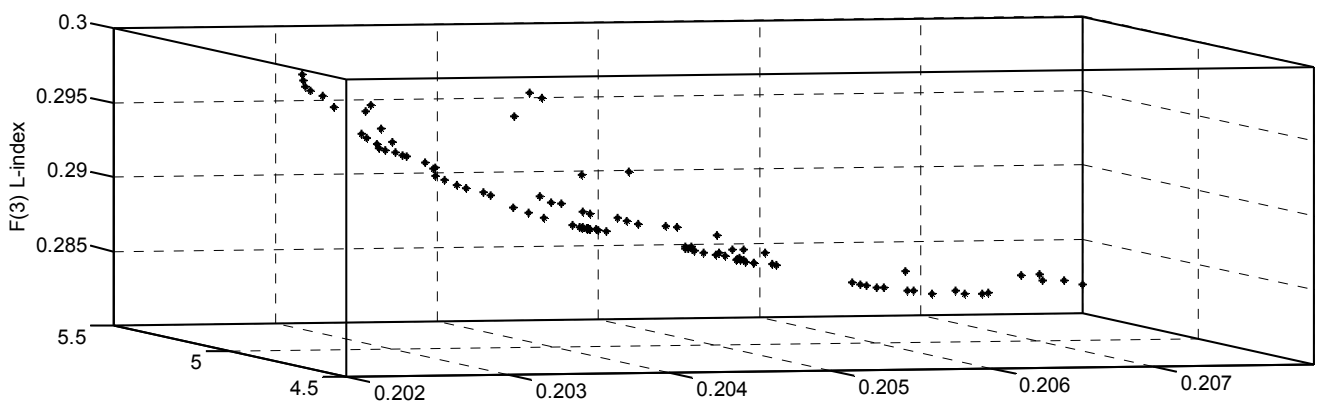

$F(2) Q(p . u)$

F(1) P (p.u)

(a)

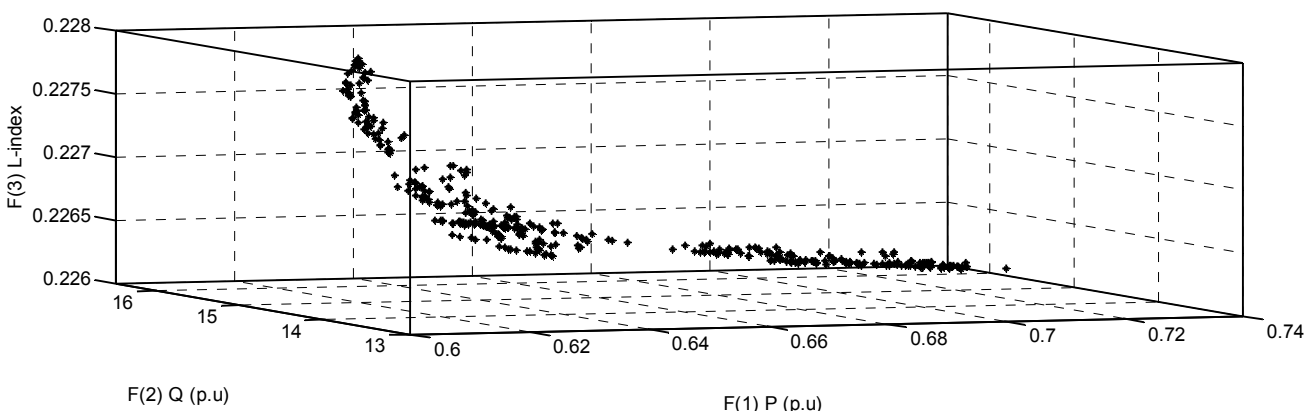

(b)

Fig. 6: Resultados de Optimización (a) TSO 2, (b) Sistema completo de 39 nodos

Paso 5: Solución del problema OPFMO. La solución fue obtenida con la técnica heurística evolutiva NSGAII, para el caso del sistema centralizado se ejecutó una sola vez, considerando 400 poblaciones y 100 generaciones, mientras que en los casos de las áreas de control se ejecutó una sola vez, con 100 poblaciones y 50 generaciones, respectivamente. La Figura 6 (a), ilustra la solución gráfica de la optimización para el área de control TSO 2, mientras que la Figura 6 (b) ilustra la optimización del sistema global de manera centralizada.

Paso 6: Selección del vector óptimo. La Tabla 2 resume los cálculos estadísticos de los valores de las variables de control obtenidas en la optimización en cada área de control y del sistema centralizado. Como se observa la varianza de los resultados para las funciones objetivos $F O_{1}$, y $F O_{3}$ es inferior a 1.0, lo que significa que la solución se encuentra en la región de los óptimos globales, mientras que la función $\mathrm{FO}_{2}$, asociada a la pérdidas de potencia reactiva para el sistema centralizado, la varianza es de 1.556 , pero que comparada con los subsistemas es mayor, lo que significa que existe una mayor dispersión en el proceso de optimización, justificado en parte porqué se utilizan más los recursos propios dentro de un área. No obstante, los valores de la varianza, son un indicativo de las soluciones en el frente de Pareto y la dispersión de los resultados no dominantes. Por lo anterior, se puede asumir que la solución multiobjetivo en cada uno de los casos es aceptable.

Tabla 2: Valores Estadísticos de la optimización sistema Centralizado vs los TSO

\begin{tabular}{|c|c|c|c|c|c|c|c|c|c|c|c|c|}
\hline & \multicolumn{3}{|c|}{ Sistema Centralizado } & \multicolumn{3}{|l|}{ TSO1 } & \multicolumn{3}{|l|}{ TSO2 } & \multicolumn{3}{|l|}{ TSO3 } \\
\hline & $\mathrm{FO}(1)$ & $\mathrm{FO}(2)$ & $\mathrm{FO}(3)$ & $\mathrm{FO}(1)$ & $\mathrm{FO}(2)$ & $\mathrm{FO}(3)$ & $\mathrm{FO}(1)$ & $\mathrm{FO}(2)$ & $\mathrm{FO}(3)$ & $\mathrm{FO}(1)$ & $\mathrm{FO}(2)$ & $\mathrm{FO}(3)$ \\
\hline Promedio & 0.646 & 14.012 & 0.227 & 0.208 & 4.766 & 0.213 & 0.204 & 4.888 & 0.289 & 0.141 & 2.706 & 0.188 \\
\hline $\mathrm{Mec}$ & 0 & 13. & 0.2 & 0.208 & 4.758 & 0.213 & 0.205 & 4.782 & 0.287 & 0.138 & 2.644 & 0.188 \\
\hline Valor & 0.582 & 12.427 & 0.226 & 0.207 & 4.750 & 0.213 & 0.202 & 4.692 & 0.284 & 0. & 2.561 & 0.184 \\
\hline Valor Máximo & 0.727 & 16.340 & 0.228 & 0.216 & 4.919 & 0.213 & 0.207 & 5.340 & 0.300 & 0.152 & 2.911 & 0.192 \\
\hline Varianza & 0.002 & 1.556 & 0.000 & 0.000 & 0.001 & 0.000 & 0.000 & 0.039 & 0.000 & 0.000 & 0.012 & 0.000 \\
\hline
\end{tabular}

Con base en la Tabla 2, se escogen los mejores candidatos de acuerdo a la ecuación (12). Para ilustrar el procedimiento, en el caso del sistema centralizado se obtuvo un valor de 1.501 (ver Tabla 3). Por tanto, este valor corresponde a la población 5 , cuyos valores óptimos $u^{*}$ calculados de las variables de control se presentan en la Tabla 4. Un procedimiento similar se sigue para el caso del sistema descentralizado, donde se integran los valores para TSO 1, TSO 2, TSO 3, presentados en la Tabla 4. 
Tabla 3: Selección de valores $u^{*}$ para el sistema centralizado

\begin{tabular}{|l|c|c|c|c|}
\hline & $\mathrm{P}(\mathrm{p} . \mathrm{u})$ & $\mathrm{Q}(\mathrm{p} . \mathrm{u})$ & L-index & $\min =\mid \Sigma\left(\right.$ Mediana $\left.\left(f_{i}\right)-\min \left(f_{i}\right)\right) \mid$ \\
\hline Menor FO (1) P & 0.582 & 12.488 & 0.228 & \\
\hline Menor FO (2) Q & 0.585 & 12.427 & 0.228 & \\
\hline Menor FO (3) L & 0.725 & 15.668 & 0.226 & \\
\hline Mediana FO & 0.650 & 13.923 & 0.227 & 1.501 \\
\hline Mediana FO - F0 (1) P & 0.068 & 1.435 & -0.002 & 1.559 \\
\hline Mediana FO - F0 (2) Q & 0.064 & 1.496 & -0.002 & 1.820 \\
\hline Mediana FO - F0 (3) L & -0.076 & -1.745 & 0.000 & \\
\hline
\end{tabular}

Paso 7: Solución del Sistema integrado

La Tabla 5 muestra el resultado de las funciones objetivos, en el cual se pueden comparar el caso base y el sistema optimizado de forma centralizado y el descentralizado. La variación de las pérdidas activas entre la propuesta descentralizada y la estrategia centralizada es de $1.69 \%$. Para el índice $L$-index, es del $0.39 \%$, valores que cumplen con el criterio definido. En cambio, las pérdidas reactivas presentan una variación del $38.45 \%$, justificado por que cada área satisface sus requerimientos, antes que tomar recursos de áreas adyacentes.

La Figura 7, presenta los flujos de potencia por los enlaces de interconexión, cuando se sustituyen los valores óptimos de las variables de control $u^{*}$ en el modelo del flujo de potencia. Como se puede observar, los flujos de potencia activa permanecen muy cercanos a los valores preestablecidos en el caso base y caso centralizado. El error máximo es del 3\%, registrado en el enlace 11-2, lo cual cumple con el criterio establecido. En contraste con la potencia reactiva se observan mayores diferencias, debido a lo mencionado en el párrafo anterior.

Finalmente, en cuanto a tiempos de cómputo, los cálculos se realizan en un equipo con las siguientes especificaciones: Toshiba Satellite T215D, AMD Athlon II Neo K125 Processor 1.7 GHz, 2.00 GB de RAM. La solución se alcanza en una sola iteración, con tiempos de cómputo así: el sistema centralizado tomó 3832 segundos mientras que sistemas TSO 1 requirió 353 segundos, TSO 2 un tiempo de 342 segundos y TSO 3 un valor de 352 segundos, para un total de 1046 segundos, es decir la 3.66 veces menos en relación al sistema integrado. Si el proceso se hace de manera distribuida en equipos diferentes el ahorro en tiempo puede alcanzar del orden de 10 veces menos.

Tabla 4: Valores óptimos $u^{*}$ de las variables de control sistema Centralizado vs Descentralizado

\begin{tabular}{|c|c|c|c|}
\hline Variable & Base & Centralizado & Descentralizado \\
\hline V1 & 1 & 1.1000 & 1.1000 \\
\hline V2 & 1 & 1.0980 & 1.1000 \\
\hline V3 & 1 & 1.0999 & 1.1000 \\
\hline V4 & 1 & 1.0999 & 1.0997 \\
\hline V5 & 1 & 1.0999 & 1.1000 \\
\hline V6 & 1 & 1.0978 & 1.0999 \\
\hline V7 & 1 & 1.1000 & 1.0997 \\
\hline V8 & 1 & 1.1000 & 1.0911 \\
\hline V9 & 1 & 1.0925 & 1.1000 \\
\hline V10 & 1 & 1.0738 & 1.0878 \\
\hline $\mathrm{T} 1$ & 1 & 1.0376 & 0.9732 \\
\hline $\mathrm{T} 2$ & 1 & 1.0796 & 0.9848 \\
\hline $\mathrm{T} 3$ & 1 & 0.9042 & 0.9718 \\
\hline $\mathrm{T} 4$ & 1 & 0.9557 & 0.0965 \\
\hline $\mathrm{SH} 1$ & 0 & 1.9921 & 0.6286 \\
\hline $\mathrm{SH} 2$ & 0 & 1.9995 & -1.6322 \\
\hline $\mathrm{SH} 3$ & 0 & -1.5237 & 1.1107 \\
\hline $\mathrm{SH} 4$ & 0 & 0.1793 & 0.9752 \\
\hline
\end{tabular}

Tabla 5: Comparación Valores FO Base, Centralizado y Descentralizado

\begin{tabular}{|c|c|c|c|}
\hline & Base & Centralizado & Descentralizado \\
\hline $\mathrm{FO}(1)$ & 0.7693 & 0.5889 & 0.5989 \\
\hline $\mathrm{FO}(2)$ & 7.4229 & 0.6737 & 0.9328 \\
\hline $\mathrm{FO}(3)$ & 0.2865 & 0.2281 & 0.2290 \\
\hline
\end{tabular}




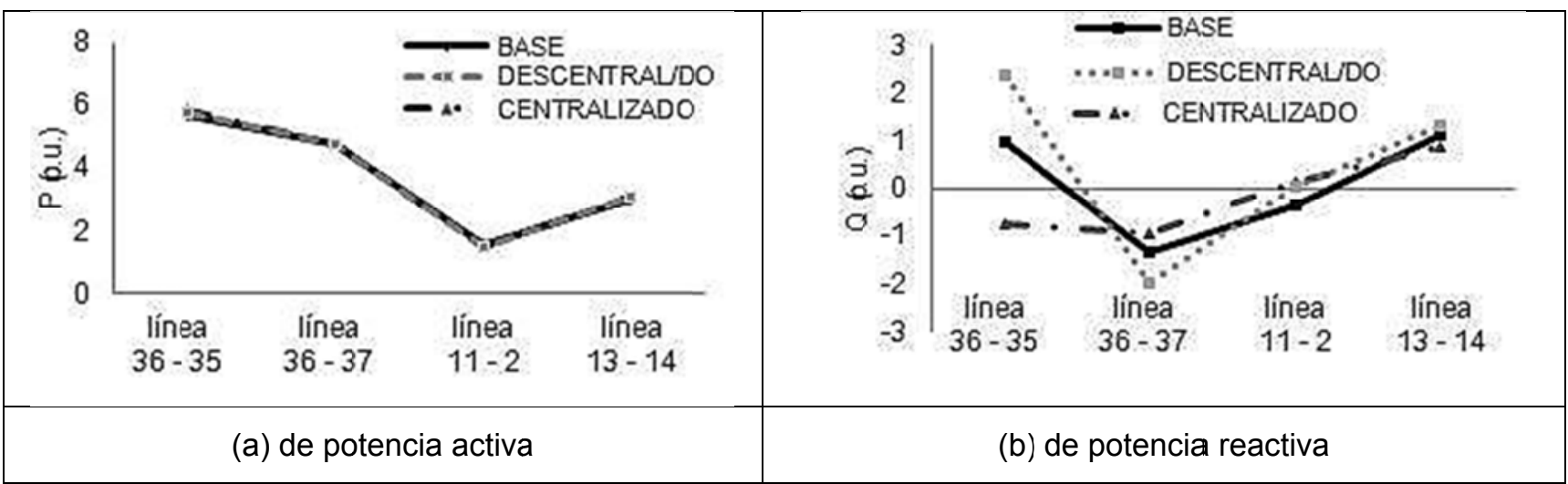

Fig. 7: Flujos de potencia por los enlaces de interconexión

Aplicación a la red de 190 nodos:

La red está compuesta por 190 nodos, 49 generadores, 257 líneas, 8 enlaces de interconexión, 96 transformadores, 20 cambiadores de tomas de transformadores, y 22 elementos de compensación estáticos. Las variables de control $\left(\left(V_{g i}\right), \quad\left(S_{i}\right), \quad\left(T_{i}\right)\right)$ están acotadas según: $-1.5 p . u \leq S_{i} \leq 1.5 p . u$ para $i=1, \ldots, 22,0.95$ p.u $\leq \operatorname{Tap}_{i} \leq 1.05$ p.u para $i=1, \ldots, 20$ y $0.95 p . u \leq V_{g i} \leq 1.05$ p.u para $i=1, \ldots, 46$.

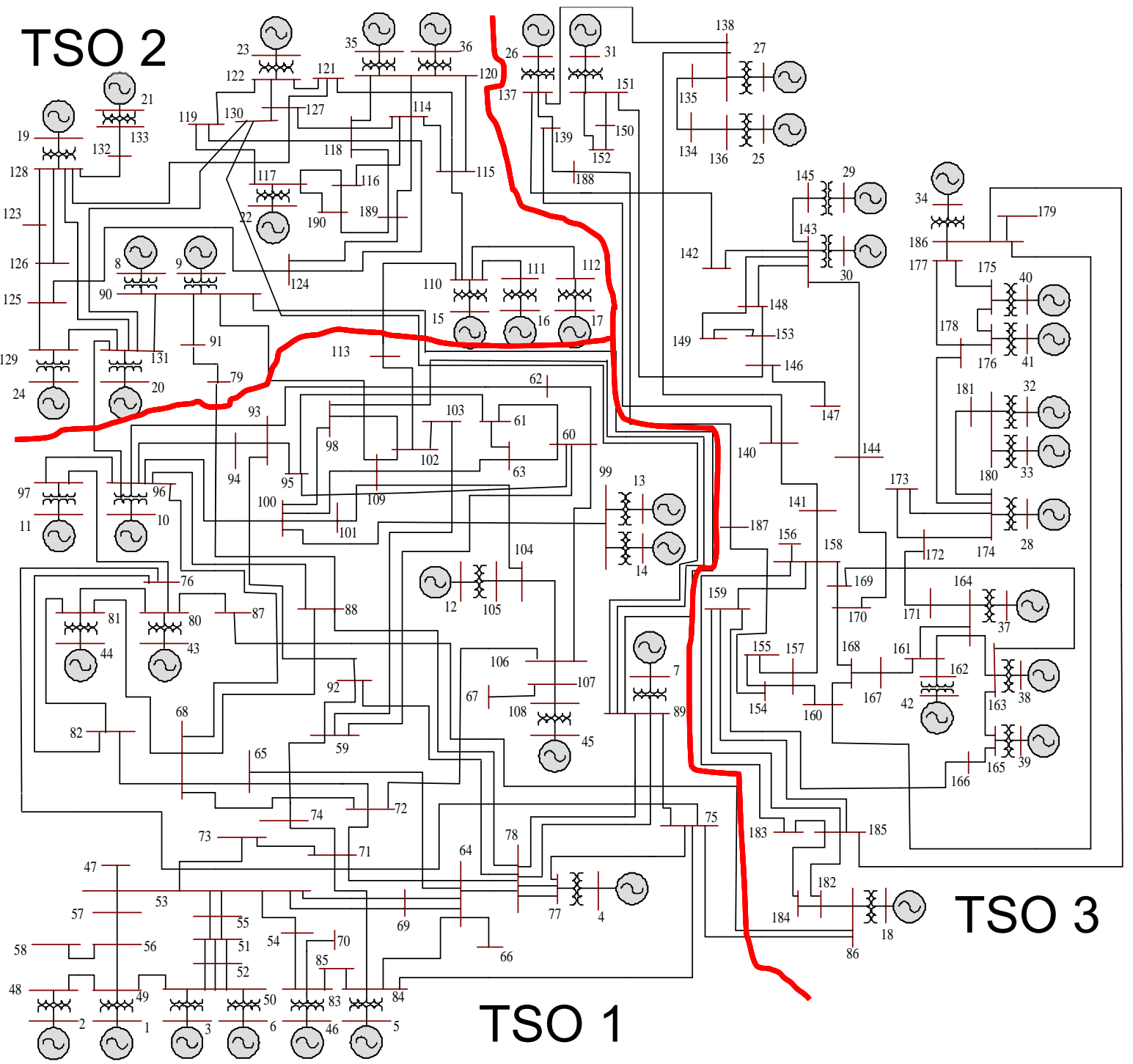

Fig. 8: Sistema de 190 nodos fraccionado en 3 TSOs 


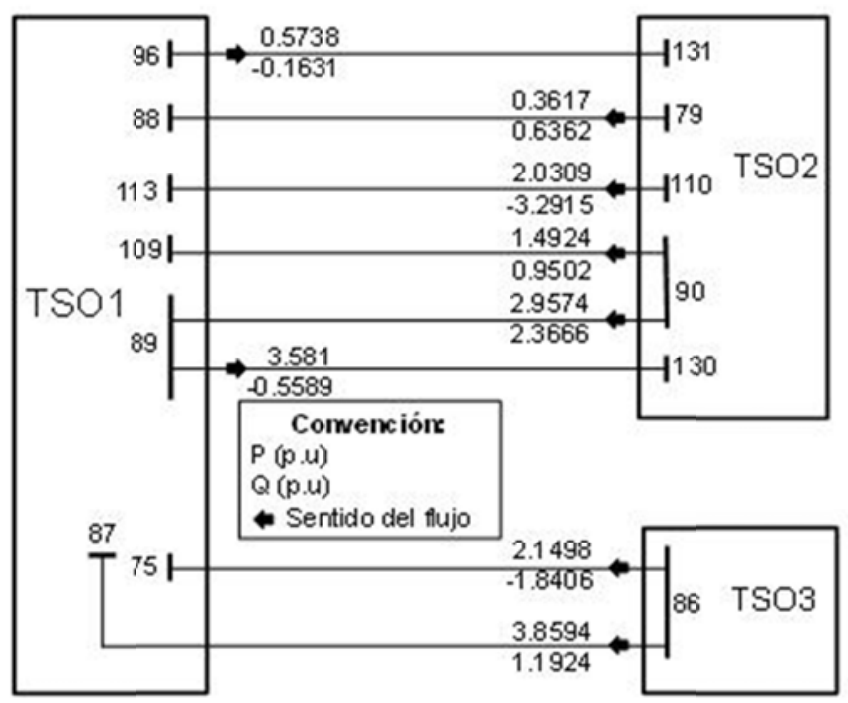

Fig. 9: Intercambios programados entre los TSOs

\section{Procedimiento metodológico}

Paso 1: Partición de la red. La red se descompone en tres sistemas, siguiendo el criterio del menor número de enlaces, tal como se ilustra en diagrama unifilar (Figura 8), donde entre TSO 1 y TSO 2 existen 6 enlaces, TSO 1 y TSO 3 dos líneas, entre TSO 2 y TSO 3 no se presentan interconexiones.

Paso 2: Se calculan los flujos de potencia y se determinan los flujos entre áreas, Figura 9.

Paso 3: Cálculo de equivalentes de red. Los equivalentes generalizados se presentan en la Tabla 6, los cuales se forman a partir de los enlaces y el resultado del flujo de potencia.

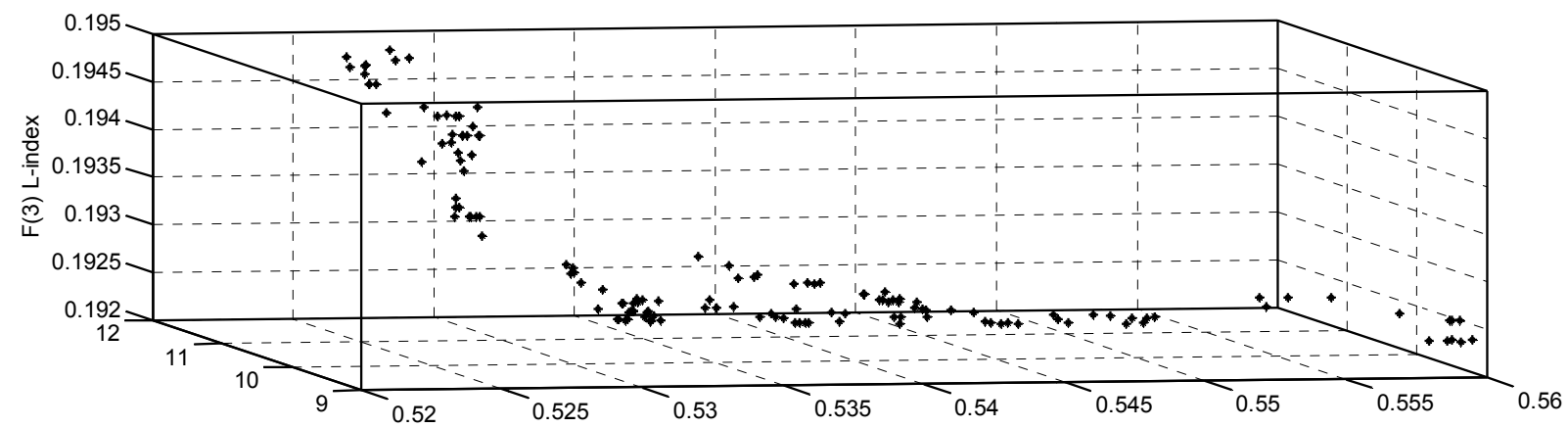

$F(2) Q(p . u)$

$F(1) P(p . u)$

(a)

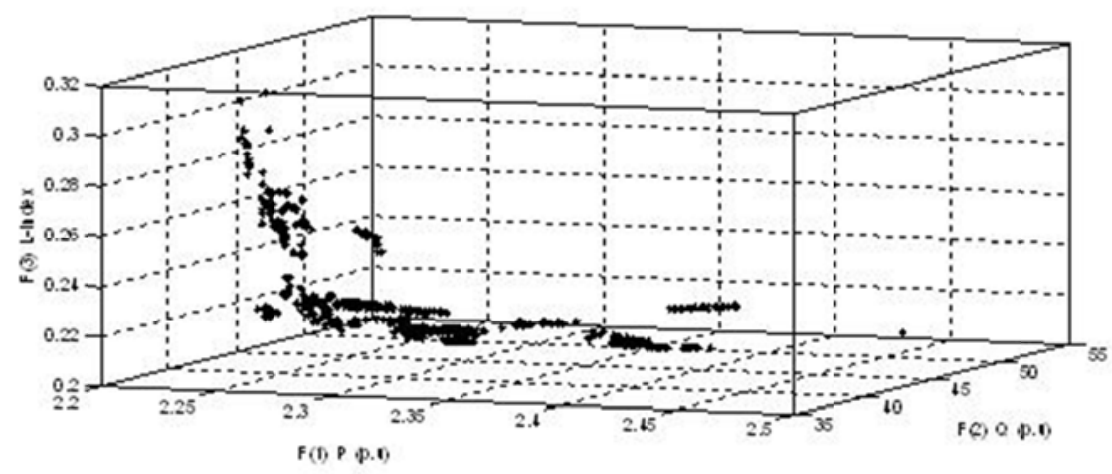

(b)

Fig. 10: Resultados de Optimización (a) TSO 3, (b) Sistema completo de 190 nodos 
Paso 4: Se construye el modelo del OPFMO. Con base en los equivalentes se actualizan los OPF para cada área. Se consideran las mismas funciones objetivos presentadas para el sistema 39 nodos. Las variables de decisión corresponden a: voltaje terminal generadores $\left(V_{g i}\right)$, total 46 ; la potencia reactiva de los elementos de compensación estáticos $\left(S_{i}\right)$, total 22; y el cambiador de tomas de transformadores $\left(T_{i}\right)$, total 20 , respectivamente.

Paso 5: Solución del problema OPFMO. Para la solución, se emplea el método NSGA-II. Para el caso del sistema centralizado se ejecutó una sola vez, considerando 500 poblaciones y 150 generaciones, mientras que en los casos de las áreas de control se ejecutó una sola vez, con 150 poblaciones y 75 generaciones, respectivamente. La Figura 10 (a), ilustra la solución de la optimización para el área de control TSO 3, mientras que la Figura 10 (b) ilustra la optimización del sistema global de manera centralizada.

Tabla 6: Equivalentes generalizados del sistema de 190 nodos

\begin{tabular}{|c|c|c|c|c|c|c|}
\hline \multirow{2}{*}{ TSO } & \multirow{2}{*}{ Nodo } & \multirow{2}{*}{ Línea } & \multicolumn{4}{|c|}{ Equivalente } \\
\hline & & & Nodo F & Tipo & $P$ (p.u) & $\mathrm{Q}$ (p.u) \\
\hline \multirow{8}{*}{1} & 75 & L $86-75$ & 86 & PV & 2,1498 & $-1,8406$ \\
\hline & 87 & L $86-87$ & 86 & PV & 3,8594 & 1,1924 \\
\hline & 88 & L $79-88$ & 79 & $\mathrm{PV}$ & 0,3617 & 0,6362 \\
\hline & 89 & L $90-89$ & 90 & $\mathrm{PV}$ & 2,9574 & $-2,2890$ \\
\hline & 89 & L $130-89$ & 130 & $P Q$ & $-3,5466$ & $-0,2798$ \\
\hline & 96 & L 131-96 & 131 & $P Q$ & $-0,5638$ & $-0,1379$ \\
\hline & 109 & L $90-109$ & 90 & $\mathrm{PV}$ & 1,4924 & 0,9502 \\
\hline & 113 & L $110-113$ & 110 & $\mathrm{PV}$ & 2,0309 & $-3,2915$ \\
\hline \multirow{6}{*}{2} & 79 & L $88-79$ & 88 & $P Q$ & $-0,3592$ & $-0,6924$ \\
\hline & 90 & L $89-90$ & 89 & $P Q$ & $-2,9574$ & 2,3666 \\
\hline & 90 & L $109-90$ & 109 & $P Q$ & $-1,4629$ & $-0,8978$ \\
\hline & 110 & L $113-110$ & 113 & $P Q$ & $-2,0167$ & $-0,1692$ \\
\hline & 130 & L $89-130$ & 89 & $\mathrm{PV}$ & 3,5810 & $-0,5589$ \\
\hline & 131 & L $96-131$ & 96 & $\mathrm{PV}$ & 0,5738 & $-0,1631$ \\
\hline \multirow{2}{*}{3} & 86 & L $75-86$ & 75 & $P Q$ & $-2,2251$ & $-1,7337$ \\
\hline & 86 & L $87-86$ & 87 & $P Q$ & $-3,7601$ & $-0,7203$ \\
\hline
\end{tabular}

Paso 6: Selección del vector óptimo. La Tabla 7 muestra los cálculos estadísticos de los valores calculados de las funciones objetivo en el proceso de optimización, con un comportamiento similar al presentado en el sistema de 39 nodos, es decir, la varianza para las funciones objetivos $F O_{1}$, y $F O_{3}$ es inferior a 1.0. Con base en la Tabla 7, se escogen los mejores candidatos de acuerdo a la ecuación (12), tal como se detalló para el sistema de 39 nodos.

Tabla 7: Valores Estadísticos de la optimización sistema Centralizado vs TSOs

\begin{tabular}{|c|c|c|c|c|c|c|c|c|c|c|c|c|}
\hline & \multicolumn{3}{|c|}{ Sistema Centralizado } & \multicolumn{3}{|c|}{ TSO1 } & \multicolumn{3}{c|}{ TSO2 } & \multicolumn{3}{c|}{ TSO3 } \\
\cline { 2 - 14 } & $\mathrm{FO}(1)$ & $\mathrm{FO}(2)$ & $\mathrm{FO}(3)$ & $\mathrm{FO}(1)$ & $\mathrm{FO}(2)$ & $\mathrm{FO}(3)$ & $\mathrm{FO}(1)$ & $\mathrm{FO}(2)$ & $\mathrm{FO}(3)$ & $\mathrm{FO}(1)$ & $\mathrm{FO}(2)$ & $\mathrm{FO}(3)$ \\
\hline Promedio & 2.302 & 41.746 & 0.228 & 1.432 & 21.509 & 0.384 & 0.513 & 11.061 & 0.361 & 0.538 & 10.053 & 0.193 \\
\hline Mediana & 2.288 & 40.457 & 0.220 & 1.400 & 20.884 & 0.381 & 0.507 & 10.252 & 0.361 & 0.535 & 9.835 & 0.193 \\
\hline Valor Mínimo & 2.215 & 37.398 & 0.217 & 1.352 & 19.512 & 0.370 & 0.444 & 7.694 & 0.360 & 0.521 & 8.753 & 0.192 \\
\hline Valor Máximo & 2.483 & 51.000 & 0.313 & 1.640 & 25.643 & 0.402 & 0.624 & 18.177 & 0.367 & 0.628 & 12.809 & 0.195 \\
\hline Varianza & 0.004 & 10.580 & 0.000 & 0.005 & 2.305 & 0.000 & 0.003 & 9.310 & 0.000 & 0.000 & 0.610 & 0.000 \\
\hline
\end{tabular}

Paso 7: Solución del Sistema integrado. La Tabla 8 presenta el valor de las funciones objetivo para el sistema centralizado el descentralizado. Como puede observase, el valor de las pérdidas activas obtenidos en la propuesta descentralizada comparada con la estrategia centralizada tiene una variación del $4.19 \%$, mientras que en las pérdidas reactivas el error es del $2.71 \%$, y en el valor del $L$-index el error calculado fue de $17.19 \%$.

Los intercambios de potencia activa y reactiva por los enlaces de interconexión entre las distintas áreas de control se ilustran en las Figuras 11 (a) y (b). Como se puede observar los flujos de potencia activa permanecen muy cercanos a los valores predefinidos. La variación máximo para este caso de simulación fue del $3.32 \%$ en el enlace $90-89$, con lo que se satisface el criterio de tolerancia. 
Tabla 8: Comparación Valores FO Base, Centralizado y Descentralizado

\begin{tabular}{|l|c|c|c|}
\hline & BASE & Centralizado & Descentralizado \\
\hline $\mathrm{FO}(1)$ & 2.3561 & 2.2880 & 2.3214 \\
\hline $\mathrm{FO}(2)$ & -66.3861 & -72.5869 & -74.5594 \\
\hline $\mathrm{FO}(3)$ & 0.2423 & 0.2181 & 0.2556 \\
\hline
\end{tabular}

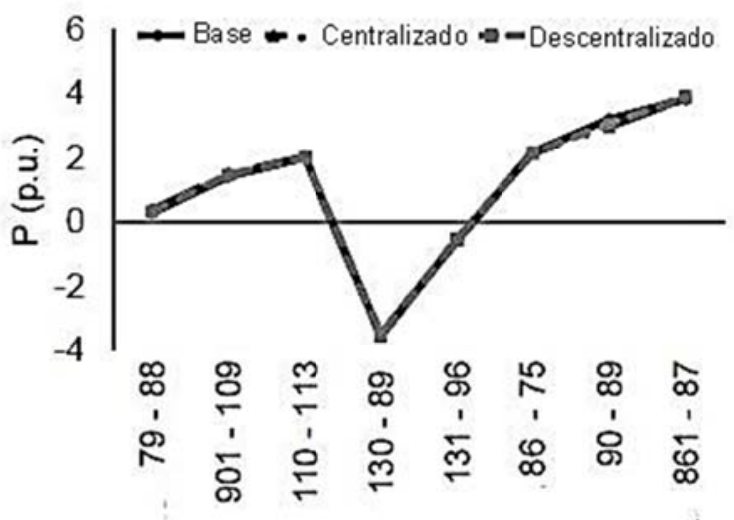

(a)

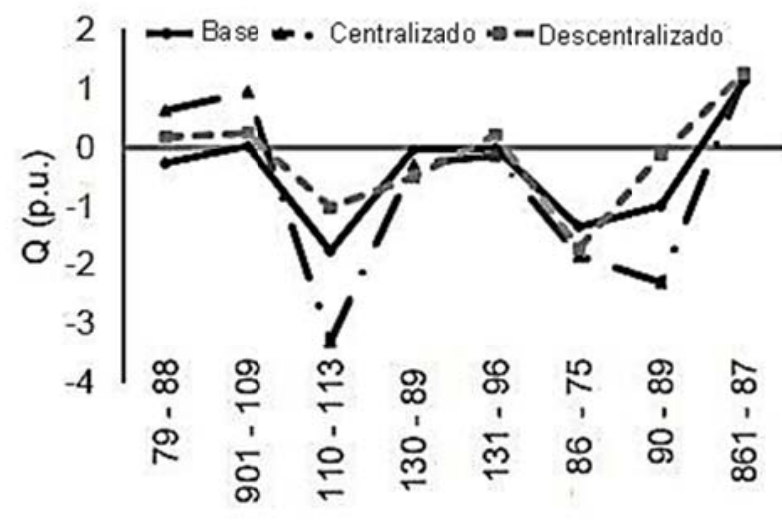

(b)

Fig. 11: Intercambios: (a) de potencia activa $\quad$ (b) de potencia reactiva

Finalmente, en cuanto a tiempos de cómputo, los cálculos se realizan en equipo con las siguientes especificaciones: HP Pavilion DV4-4063 LA - Laptop Intel Corel i5-2410M 2.3GHz, 640GB, RAM 4GB. EI proceso se ejecuta en modo secuencial, la optimización de cada subsistema TSO 1 requiere 128 segundos, TSO tomó 123 segundos y TSO 3 un tiempo de 192 segundos para un total de 443.28 segundos que equivale a 6.5 veces menos que el tiempo que toma la optimización de manera centralizada 2888 segundos. $\mathrm{Si}$ el proceso se hace de manera distribuida, en equipos diferentes, el ahorro en tiempo puede alcanzar el orden de 15 veces menos.

\section{CONCLUSIONES}

La metodología de optimización propuesta en este trabajo brinda la posibilidad de operar la red de energía eléctrica de una forma fraccionada y autónoma, al proponer una estrategia descentralizada, y más ágil al asumir los intercambios entre las diferentes áreas como fruto de un despacho económico, lo que simplifica los equivalentes de red y permite convertir las variables de frontera en constantes durante todo la optimización. La metodología explotó los atributos de la descomposición tanto de la red eléctrica como del problema de optimización, obteniéndose reducción de los tiempos de cálculo a más de 5 veces, al involucrar menos variables en el proceso y con menor requerimiento de información operativa entre los operadores de red.

Se consideró la seguridad de la red, al tener en cuenta el índice de estabilidad, L-index, como función objetivo, a parte de las pérdidas activas y reactivas, con lo cual se determinaron los rangos admisibles de las variables más importantes de la red.

En la solución del problema de optimización multiobjetivo, se logró la convergencia a puntos de operación adecuados en la región óptima en el proceso de optimización, de una forma muy rápida al utilizar una técnica heurística evolutiva, como NSGA-II.

La utilización estadística del valor mínimo y la mediana del conjunto de soluciones permitió seleccionar los valores más adecuados de ponderación de las funciones objetivos dentro de los valores admisibles dados por un frente de Pareto. En la validación de la metodología donde se comparan intercambios, pérdidas y tiempos de cálculo con los resultados obtenidos al aplicar una estrategia centralizada a la misma red, muestran que es posible abordar la operación de grandes sistemas de potencia con múltiples operadores de red de manera descentralizada.

En ambos casos de aplicación de la metodología, se encontraron resultados coherentes con los objetivos propuestos. 


\section{REFERENCIAS}

Amarnath R. V., y Ramana, N. V., State of Art in Optimal power Flow solution methodologies,. Journal of Theoretical and applied information technology, 30, 128-154 (2011).

Arnold, M., Knopfli, S., Andersson, G., Improvement of OPF Decomposition Methods Applied to Multi-Area Power Systems,. Power Tech, 2007 IEEE Lausanne, 1308,1313, (2007).

Biskas P. N. y Bakirtzis A. G, Decentralized OPF of Large multi-area power system. IEEE Proceedings on Generation Transmission \& Distribution, 153, 99-105, 12 de Enero (2006).

Borges, D., Pérez, I. y León, V. Compensación de potencia reactiva en sistemas desbalanceados utilizando algoritmos genéticos. Ingeniare. Revista Chilena de Ingeniería, 20 (3), 284-292, (2012).

Coello Coello C., Lamont C., y Van Veldhuizen D, Evolutionary Algorithms for Solving Multi-objective Problems. Springer,. (2007).

Dantzig G. B. y Wolfe P, Decomposition principle for linear programs. Operations Research, 8(1), $101-111$. (1960).

Deb, K., Agrawal, S, Pratap, A., y Meyarivan, T., A fast and elitist multiobjective genetic algorithm: NSGA-II,. IEEE Trans. Evolutionary Computation, 6(2), 182-197 (2002).

Deb K., Zope P., Jain A., Distributed Computing of Pareto-Optimal Solutions Using Multi-Objective Evolutionary Algorithms. Report No. 2002008, Kanpur Genetic Algorithms Laboratory, Indian Institute of Technology Kanpur. (en línea), (2002), http://www.iitk.ac.in/kangal/pub.htm. Acceso : 21 de Agosto (2011)

FERC, Federal Energy Regulatory Commission of the United States of America, Staff Report, Principles for eficient and reliable reactive power supply and consumption,. Docket. No. AD05-1-000, February 4 (2005).

González, X., Ramírez J. M., Medina M., Reactive power handling by a multi-objective formulation, North American Power Symposium (NAPS), 1-5, 4 a 6 de Agosto (2011)

Kessel P. y Glavitsch, H., Estimating the Voltage Stability of a Power System,. Power Engineering Review, IEEE PER-6 (7), 72 (1986).

Kim B. H. y Baldick R, Coarse-grained distributed optimal power flow, IEEE Transactions on Power Systems, 12(2), 932-939. (1997)

López, J., Determinación y Localización de Zonas de Mayor Energía Modal en Sistemas Eléctricos de Potencia,. Ms.C. Tesis, CINVESTAV Unidad de Guadalajara, Enero (2013).

Marinakis A., Glavic M. y Van Cutsem T., Control of phase shifting transformers by multiple transmission system operators. Power Tech, 2007 IEEE Lausanne, 119-124, 1 a 5 de Julio (2007).

Mendoza, J. y Rojas, F. Restauración de servicio multiobjetivo en redes de distribución utilizando NSGA-Il. Ingeniare. Revista Chilena de Ingeniería, 17(3), 337-346. (2009)

Mousavi O. A. y Cherkaoui, R., Literature Survay on Fundamental Issue of Voltage and Reactive Power control,. EPF Lausanne Power System Group, Junio (2011).

Nogales F, Prieto F, y Conejo A, A decomposition methodology applied to the multi-area optimal power flow problem. Annals of Operations Research, 120, 99-116, (2003)

Padiyar K. R, Power System Dynamics. Stability and Control. Indian Institute of Science, B., John Wiley \& Sons (Asia): Singapore. (1996).

Phulpin, Y, Begovic, M. y Ernst, D., Coordination of voltage control in a power system operated by multiple transmission utilities,. In REP Symposium, Buzios, 1-8, 1 a 6 de Agosto (2010).

Phulpin, Y. D., Coordination of reactive power scheduling in a multi-area power system operated by independent utilities,. PhD. Thesis, Georgia Institute of Technology (2009). 
Srinivas, N. y Deb, K., Multiobjective Optimization Using Nondominated Sorting in Genetic Algorithms,. Evolutionary Computation 2(3), 221-248 (1994).

Suganyadevia, M. V. y Babulal, C. K., Estimating of loadability margin of a power system by comparing Voltage Stability Indices,. International Conference on Control, Automation, Communication and Energy Conservation, 2009. INCACEC 2009, 1-4, Erode, India 4 a 6 de Junio (2009).

Swiss Federal Office of Energy (SFOE), Report on the blackout in Italy on 28 September 2003 (2003).

Venkatesh, B., Sadasivam, G. y Khan, G., A new optimal reactive power scheduling method for loss minimization and voltage stability margin maximization using successive multi-objective fuzzy LP technique, . Power Systems, IEEE Transactions on 15 (2), 844-851 Mayo (2000).

X. Wang, Y. H. Song y Q. Lu, Lagrangian decomposition approach to active power congestion management across interconnected regions. IEE Proc. Generation Transmission \& Distribution, 148(5), 497 - 503, September (2001). 
